\title{
BEHAVIOR OF SILVER AND GOLD NANOPARTICLES IN SIMULATED BIOLOGICAL FLUIDS: NEEDS AND CHALLENGES
}

\author{
ZAMFIRA DINCA ${ }^{\mathrm{a}}$, TEODORA MOCAN ${ }^{\mathrm{b}, \mathrm{c}}$, \\ LACRIMIOARA SENILA ${ }^{a}$, OANA CADAR ${ }^{\mathrm{a}, *}$
}

\begin{abstract}
Nanotechnology is an attractive and challenging science focused on the materials at nanoscale level and their employment in a wide variety of applications. Its applications in (nano)medicine are constrained due to the main concerns in understanding and predicting the behavior of nanoparticles (NPs) in complex biological fluids. The properties of NPs can be strongly influenced by the surrounding conditions, such as $\mathrm{pH}$ and composition, in terms of aggregation status, chemical reactivity and surface chemistry. In this regard, this review intents to provide some insight into the performance of complex biological fluids associated with nanomaterials. In particular, the behavior of AgNPs and AuNPs in simulated biological fluids that must be addressed to develop an appropriate system for medical applications, from a chemical perspective, mostly related to the physicochemical characteristics of NPs and simulated biological fluids and their possible interactions (mechanisms) was considered. Furthermore, the fate of AgNPs and AuNPs during the gastrointestinal transit from an in vivo experimental perspective, as well as their potential impact on gut microbiota was also systematized.
\end{abstract}

Keywords: silver, gold, nanoparticle, simulated biological fluid, gastrointestinal microbiota

a INCDO-INOE 2000, Research Institute for Analytical Instrumentation, 67 Donath Street, RO400293, Cluj-Napoca, Romania

b Iuliu Hatieganu University of Medicine and Pharmacy, Physiology Department, 1 Clinicilor Street, RO-40006, Cluj-Napoca, Romania

c Regional Institute of Gastroenterology and Hepatology, Nanomedicine Department, 5 Constanta Street, RO-400158, Cluj-Napoca, Romania

* Corresponding author: oana.cadar@icia.ro 


\section{INTRODUCTION}

The metal oxide and metal-based nanomaterials can be considered a fast-growing area due to their wide applications, including (nano)medicine, where nanotechnology has shown a lot of potential in therapeutics and diagnostics. Additionally, Ag and Au are prospective candidates for diagnosis and drug delivery [1]. The physico-chemical characteristics of nanomaterials (i.e. small size, large surface area to volume ratio and surface energy) can result in toxicological effects, when nanomaterials enter into biological systems through the adsorption and dissolution, modifying the structure of various macromolecules.

Despite a general acceptance that NPs are persistent as bulk, small nanoparticles (NPs) are normally slightly unstable and short-lived due to their high surface energy. Consequently, there is an increasing concern about the risk of possible pollution and diseases caused by nanomaterials during manufacturing or use. In aqueous solutions, this type of NPs can aggregate, dissolve or remain suspended as single particles depending on the environmental conditions such as $\mathrm{pH}$, ionic strength, temperature, presence of organic matter, etc. [2].

The physico-chemical characteristics of nanomaterials under various conditions are a critical step in order to understand the possible (toxic) effect on the human health and environment. Though, the toxic effect can be higher for nanomaterials, since they can easily penetrate the cell membrane and deliver metal ions in high quantities because of their high surface area [3]. Among various physico-chemical properties of the NPs, the size plays a major role on their solubility in biological media. In this regard, the critical size of NPs is considered $20-30 \mathrm{~nm}$, where they exhibit distinct properties which are absent in case of larger particles with the same chemical composition. The particles tend to reduce their surface energy due to the Gibbs-Thomson effect and consequently, the smaller NPs which own a high surface energy are more reactive and dissolve faster [4].

The full understanding of the behavior of NPs in biological fluids helps to check their biological effects and supports the design of suitable NPs for the desired goal. The NPs used for implantation purpose needs to be biopersistent, the NPs designed to release ions should be biodegradable, while the NPs administered as contrast agents are preferred to cleared out rather than accumulating inside the body [5]. The in vivo experimental data for NPs ingestion should be also considered, in light of elucidating their impact on the gastrointestinal microbiota. To assess the interaction of NPs with the gut microbiome, a better understanding of the ecology, physiology of microorganisms and physico-chemical properties of NPs is needed. The characteristics of NPs 
(size, surface chemistry and charge), $\mathrm{pH}$, type of meal and their nutritional characteristics, enzymatic activities, mucosal secretion or host microbiota can affect the fate and bioavailability of NPs during the gastrointestinal transit [6].

This review systematizes the key factors of AgNPs and AuNPs and simulated biological fluids that must be considered to develop a suitable system for medical applications, mainly: (i) physico-chemical characteristics of NPs and simulated biological fluids; (ii) possible interactions between NPs and simulated biological fluids, (iii) the control and change of these characteristics for biomedical applications and (iv) the potential impact of NPs to the gastrointestinal microbiota.

\section{RESULTS AND DISCUSSION}

\section{Behavior of nanoparticles in simulated biological fluids and their impact on the gut microbiota}

The study of dissolution behavior of NPs offers valuable information on their interactions with the biological and environmental surroundings. If the particles release ions at a fast rate, their short-term toxic effect will be similar to that of the dissolved ions, while if the particles release ions at a slow rate, those particles may cause long-term effects in humans [7, 8]. Therefore, the dissolution rate of NPs is a key factor for the assessment of their possible toxic effects. The released ions join in competing reactions, their mobility is high and their concentration is low, the system outlying from the saturation point and, consequently, the NPs tend towards complete dissolution. However, if during the dissolution process, the concentration of available ions brings the system to saturation, the ions will dissolve more slowly.

In terms of in vivo experiments, the behavior of NPs in the gastrointestinal environment must also study in the presence of more than 100 trillion microorganisms of human commensal microbes [9, 10]. The human gastrointestinal tract (GIT) is colonized by various species of bacteria, viruses, fungi, archaea and protozoans termed gut microbiota [11]. About $90 \%$ of bacteria are belonging to the phyla Firmicutes and Bacteroidetes, while other $10 \%$ species are from the phyla Proteobacteria, Verrucomicrobia, Actinobacteria, Fusobacteria and Cyanobacteria [12]. The microbial communities play an essential role in the development and differentiation of the intestinal epithelium, contribute to digestion and fermentation of indigestible polysaccharides, produce vitamins, support the host immune defense against pathogens [13] and play an important role in maintaining long-term intestinal hemostasis [14]. The studies on germ-free mice and piglets showed that the 
absence of any intestinal flora, leads to underdevelopment of intestinal epithelium in mice or aberrant epithelial surface which causes passage of harmful luminal microorganisms, microbial toxins or foreign antigens [15].

Gut microbiome is a dynamic system that changes depending on factors such as the age, the dietary habits, [16] geographic environment [17] the use of drugs including antibiotics $[11,18]$ or in response to the disease [19]. Due to the interaction with the environment and external factors, the human microbiota can register imbalances named dysbiosis [20]. The dysbiosis occurs when Firmicutes/Bacteroidetes (F/B) ratio, which indicates the health status of the GIT becomes unbalanced [21], and has been associated with the development of inflammatory bowel disease (IBD) [22], irritable bowel syndrome (IBS) [23], cardiovascular diseases [24], neurodegenerative disorders [25], kidney stones [26] or metabolic diseases including obesity and diabetes [27]. Many causes of dysbiosis have been associated with various products and supplements containing NPs. The widespread application of NPs increases the direct human ingestion and access to the host GIT and microbiota [28]. It has been shown that AgNPs accumulate in the stomach, duodenum, ileum, jejunum and colon, with potential negative impact on the gut microbiome and human health [28]. In this context, understanding the microbial communities and their behavior in the presence of NPs is essential to fully assess the potential impact on the human body [29].

\section{Silver nanoparticles}

Due to their antibacterial properties, the AgNPs are used in many scientific designs and developments, they enter into the human body or the environment either accidentally or purposefully. The brought biological effects or potential toxicity of AgNPs are highly dependent on their colloidal (aggregation) and chemical (interaction with protein) stability [30].

The specific bactericidal mechanism of $\mathrm{Ag}$ is not fully understood, but could result from the interaction of $\mathrm{Ag}^{+}$ions with enzymes or generation of free radicals that causes damage bacteria cell membranes [31, 32]. The U.S. Environmental Protection Agency (EPA) suggests a reference dose (RfD) of $0.005 \mathrm{mg} / \mathrm{kg} / \mathrm{day} \mathrm{Ag}$, a value based on the risk for argyria, a gray or bluegray discoloring of the skin [33]. The exposure of AgNPs can take place from drinking water, through inhalation or direct skin or organ contact. However, there are still large gaps in understanding AgNPs toxicity [34]. The changes of particle size, surface and charge, purposefully or due to the processing affect the toxicity towards living organisms. In this regard, well-designed in vitro and in vivo studies were developed to characterize the human exposure to NPs in terms of physico-chemical nature, aggregation state, particle size, functionalization, concentration and potential risk to humans [35]. 
Generally, the analytical techniques for Ag determination are atomic absorption spectrometry (AAS), inductively coupled plasma mass spectrometry (ICP-MS) and inductively coupled plasma optical emission spectrometry (ICPOES), while the separation of AgNPs and $\mathrm{Ag}^{+}$ions is typically carried-out by centrifugation, dialysis or ultrafiltration. The above-mentioned techniques before the measurements demand the separation of $\mathrm{Ag}^{+}$ions from the AgNPs, because they detect total $\mathrm{Ag}$ content and not its oxidation state. Also, the above-mentioned techniques cannot distinguish between AgNPs and $\mathrm{AgCl}$, measuring only the dissolved forms of $\mathrm{Ag}[32,36]$. So, it is a critical step to develop methods in order to differentiate between the toxicities of the metal NPs, the dissolved metal ions and their corresponding ion complexes [32]. Although, several studies on the dissolution kinetics of AgNPs were reported, they are difficult to be compared due to: (i) the different physico-chemical properties of NPs (size, charge, surface functionalization) which strongly influence the dissolution behavior, (ii) the use of various, complex dissolution media and (iii) the released $\mathrm{Ag}^{+}$ions in solution are quickly precipitated as $\mathrm{AgCl}$ as separate particles, seemingly not on the surface of the initial Ag particles; also, the solubility product $(\mathrm{AgCl})$ and the redox potential of $\mathrm{Ag}^{+}$ions strongly depend on the initial (total) concentration [36].

The investigation of AgNPs coated with thick and sub-nanometer range surface layers, in terms of aggregation, sedimentation and dissolution in two biological fluids (artificial interstitial and lysosomal fluids) was conducted to better understand their behavior after inhalation. After introducing into high ionic strength biological fluids, the AgNPs formed aggregates and settled out, independents of surface modification. Furthermore, single NPs and aggregated coexisted in both studied biological media [2].

Treatment of textiles with AgNPs is one of the most commercialized applications due to their anti-bacterial, anti-static and mothproofing properties [37]. Beside the typical study of Ag release into the environment, recently, the researchers have begun to emphasis on the release of $\mathrm{Ag}$ into biological fluids and the potential exposure from contact with textile products. In this regard, the used fiber treatment technique was a more dominant exposure factor comparing to physiological properties of artificial saliva or sweat (composition, $\mathrm{pH}$ and temperature) [38]. Regarding a possible acting mechanism, the textiles treated with AgNPs release primarily $\mathrm{Ag}$ in ionic form when in contact with biological fluids such as sweat, saliva and urine. Although, the product must release sufficient $\mathrm{Ag}^{+}$ions to assure efficiency, while the release rate should not pose a dermal or environmental exposure hazard [34]. Hence, the influence of fiber treatment technique is important because, in the absence of acceptable limits for dermal exposure to $\mathrm{Ag}$, it indicates that the obtaining techniques could be tailored to control $\mathrm{Ag}^{+}$ion release in order to ensure the product efficacy and diminish the human and environmental exposure [38]. 
The skin surface fluids and barrier integrity are relevant characteristics when assessing the potential exposure from textiles. Several studies reported that from the physico-chemical characteristics of artificial sweat, $\mathrm{NaCl}$ and $\mathrm{pH}$ have a minor influence on $\mathrm{Ag}$ release (ionic and particulate) from a textile [37, 39]; furthermore, $\mathrm{Ag}$ is easily absorbed from suspension through abraded skin compared to intact skin [40].

The Ag release increased with the increase of the exposure time, with a quite different behavior under different $\mathrm{pH}$ values, i.e. sub-micrometer particles and ionic forms in acid perspiration solution, $\mathrm{SL} 1(\mathrm{pH} \approx 3)$, mostly $\mathrm{Ag}^{+}$ ions in alkaline perspiration solution, $\mathrm{SL} 2(\mathrm{pH} \approx 8)$ and NPs in salt perspiration solution SL3 $(\mathrm{pH} \approx 8)$, despite partial aggregation. Moreover, the acidic $\mathrm{pH}$ caused the fastest release rate and greatest $\mathrm{Ag}$ release [37]. A possible release mechanism was suggested in each case, as follows: $(i)$ acid SL-1, the relatively stable dissolved $\mathrm{Ag}$ resulted from the aggregation of the particles, while some of them dissolved into the $\mathrm{Ag}^{+}$ions, (ii) alkaline SL-2, the surface oxide increased, the unstable $\mathrm{AgO}$ being transformed into $\mathrm{Ag}^{+}$ions in the solution and (iii) SL-3, the AgNPs in neutral aqueous solution is relatively stable and only partially converted into an ionic state [37]. The exposure risk of AgNPs was higher in the simulated salt conditions, which indicates that AgNPs may release into in the salt perspiration condition and could further enter the body and the environment.

Generally, the citrate-coated AgNPs are the most popular Ag colloids for various applications, the citrate acting as reducing agent and a stabilizer by decorating the negatively charged particles [41]. In this regard, the Ag dissolution in cell culture medium (DMEM) measured using a simple localized surface plasmon resonance (LSPR) UV-visible absorbance measurement, highlighted a fractional dissolution of different polymer-coated AgNPs (citrate, dextran, poly(vinylpyrrolidone)/ PVP and poly(ethyleneglycol)/ PEG). The dissolution increased at high AgNPs concentrations and PEG coatings, but decreased considerably in case of higher molecular weight coatings. The used absorbance method was recommended as optimal to measure the amount of $\mathrm{Ag}$ remained in AgNPs, considering that AgCl do not display a LPSR absorbance [32].

The dissolution kinetics of AgNPs (300 and $600 \mu \mathrm{g} / \mathrm{L}$ ) in quarterstrength Hoagland medium revealed a directly relationship between the particle size and concentration, and the Ag release rate. As expected, smaller particles were found to dissolve faster and the used kinetic model using hard sphere theory to define the $\mathrm{Ag}^{+}$ions release fitted well the experimental data [41]. Furthermore, the AgNPs dissolved faster in biological media with low $\mathrm{pH}$ (gastric acid) comparing to pseudo-extracellular fluid and strongly interacted 
with thiol- and selenide-containing biomolecules [42]. Loza et al. also confirmed that thiol-containing compounds (cysteine) can block the particle surface and prevent the dissolution, while the organic molecules complexate the $\mathrm{Ag}^{+}$ions and accelerate the dissolution. Reducing sugars (glucose) decreased, but do not totally avoid the oxidative dissolution of $\mathrm{Ag}$ [36].

A very interesting study reported that AgNPs did not present any bactericidal effect when $E$. coli were cultivated under strictly anaerobic conditions, meaning no dissolution of the NPs taken place [43]. The surface coatings usually improve the dispersion of NPs, but can also display an acute effect on the solubility of NPsy. For example, when AgNPs are coated with 11-mercaptoundecanoic acid, a thiolated ligand with stronger affinity to the metal surface than $\mathrm{Na}$ citrate, no significant decrease in the absorption intensity up to $48 \mathrm{~h}$ incubation was remarked [44]. The in vivo and in vitro behavior of NPs suspended in a fluid can be influenced through the dispersion state or interactions at the surface, resulting in the formation of coatings around NPs.

From the product that contains, AgNPs reach the human body, most often, by oral ingestion and pass through the GIT. The biochemical composition of mouth, stomach and intestine environment can affect the AgNPs bioavailability and toxicological properties [45]. Studies performed on rats and mice using 9 and $10 \mathrm{mg} / \mathrm{kg}$ bw/d AgNPs with 28 days exposure time and 14, 20 and 110 $\mathrm{nm}$ particle size, revealed no significant alteration in the caecal microbiota composition [46, 47]. A longer exposure of rats for 13 weeks at 9,18 and $36 \mathrm{mg} / \mathrm{kg}$ bw/d concentration with 10,75 and $110 \mathrm{~nm}$ size AgNPs showed an increase of Bacteroidetes species and pathogenic gram-negative bacteria, and decrease of Firmicutes, Lactobacillus and Bifidobacterium in ileac microbial populations [48]. Therefore, the doses of exposure and particle sizes are influencing factors on host gene expression [48]. The intestinal microbiota displayed to $1 \mu \mathrm{g} / \mathrm{mL}$ AgNPs in vitro batch fermentation models inoculated with human faecal matter and the probiotic Bacillus subtilis (BS) revealed that the core bacterial community was not affected but the relative abundance of Firmicutes species was increased and Bacteroidetes species decreased instead. Without BS, single treated AgNPs induces functional differences in cell motility, translation, transport, and xenobiotics degradation [49].

A shifted Firmicutes/Bacteroidetes (F/B) ratio has been observed in the rats and mice microbiota at 2.5 or $3.6 \mathrm{mg} / \mathrm{kg}$ bw/d AgNPs for 7 days or 2 weeks $[28,50]$ leading to the conclusion that the shape of AgNPs may cause an alteration of the microbiota [50]. A 28 days experiment with mice orally exposed to food pellets supplemented with 0,460 or $4600 \mathrm{ppb}$ AgNPs ( $\mu \mathrm{g} \mathrm{Ag} \mathrm{NP/kg} \mathrm{pellet)} \mathrm{revealed} \mathrm{microbial} \mathrm{alterations} \mathrm{in} \mathrm{the} \mathrm{gut} \mathrm{and} \mathrm{a} \mathrm{shift} \mathrm{in}$ the F/B ratio in the fecal microbiota [51]. It was showed that AgNPs treatment reduced Bacteroides species and increased the Coprococcus, Lactobacillus 
and Blautia taxons [51]. Disturbance in these bacterial populations were observed in the case of obese individuals [52]. Interestingly, the identical exposure of mice to the AgNPs supplemented pellets aged during 4 or 8 months has small or no effect on the F/B ratio due to the $\mathrm{Ag}$ sulfidation occurring in this matrix [51]. Another in vitro investigation performed with 33 bacterial strains isolated from a healthy human donor stool has evaluated the effects of AgNPs on the microbial communities [53]. The exposure of the bacterial community to 0 to $200 \mathrm{mg} / \mathrm{L}$ AgNPs concentrations for $48 \mathrm{~h}$ determined a negative impact on the microbial consortium with significant reduction in culture-generated gas production and changes in fatty acid methyl ester profiles [53]. Negative effects were observed in the community structure even at the lowest concentrations, with increasing abundance of species such as Raoultella sp. and of $E$. coli which are involved in different pathology or which facilitates the growth of pathogenic bacteria [53].

Even if the complete mechanisms of AgNPs interaction with the intestinal microbiota is not well understood, some studies suggested that alterations of the microbial taxons are explained by the release of $\mathrm{Ag}^{+}$ions [54], composition, size, shape surface charge, capping agent used (poly(diallyldimethylammonium)coated AgNPs, biogenic-Ag and oleate-AgNPs [55] or by forming reactive oxygen species [56].

\section{Gold nanoparticles}

The growing interest in AuNPs due to their singular physico-chemical properties, high biocompatibility and opportunity to tailor the size and shape resulted in many applications in the fields of catalysis, imaging, nanophotonics, nanomagnetic, nanoelectronic devices, biosensors, chemical sensors and drug delivery. Among all the properties, the size stability and a good dispersion are required for a high therapeutical performance [57].

The physico-chemical properties of the orally ingested AuNPs are strongly influenced by the composition ( $\mathrm{pH}$, salts and enzymes) of gastrointestinal fluid, resulting in unexpected biological outcomes. Recently, some studies reported the biodistribution and accumulation of AuNPs in cell lines and murine models, and the factors that influence their toxicity have been linked to their surface charge and functionalization, size and shape $[8,58]$. The dissolution behavior of AuNPs in simulated biological systems (i.e. artificial alveolar fluid, weak hydrochloric acid for gastric conditions, ammonium acetate solution for neutral lung fluids) depended mainly on the physico-chemical characteristics of both particles and simulated model ( $\mathrm{pH}$ and ionic strength) [59]. 
Beside the characteristics of simulated biological media, the surface charge and functionalization, size and shape of AuNPs can noticeably influence the dissolution of AuNPs [8,60]. In this regard, the dissolution kinetics of uncoated uncoated citrate-stabilized AuNPs (cit-AuNPs), polyethylene glycol (PEG)-coated AuNPs functionalized with carboxylic acid (COOH-AuNPs) and amine ( $\mathrm{NH}_{2}$-AuNPs) groups were investigated in Gamble's fluid (GF) and phagolysosomal fluid (PSF), gastric fluid (GIF) and intestinal fluid (IF) and blood plasma (BP). Generally, the dissolution of AuNPs in acidic media (PSF and GIF) was greater than that in neutral or alkaline media (Gamble's fluid, blood plasma and intestinal fluids). The cit-AuNPs tended to aggregate resulting in low surface area to volume ratio and low dissolution, while the PEG-coated AuNPs were less disposed to aggregation and displayed higher dissolution. Some possible explanations could be various ligand-promoted processes and the high stability of the cit-AuNPs [8]. Therefore, cit-AuNPs may cause long-term health effects because they are more (bio)durable and show high biopersistence, meaning these NPs might resist chemical or biochemical alteration in biological systems.

Nowadays, the use of AuNPs as therapeutic agents to treat many autoimmune, infectious and neoplastic diseases [61] is of main interest, but the effect of administered AuNPs on gut microbiota is very less studied and not well understood. However, one study has shown that AuNPs with small particle size $(5 \mathrm{~nm})$ coated by different agents such as citrate or PVP has proved anti-inflammatory effect in experimental mouse colitis, reducing the cell surface lipopolysaccharide (PLS) receptor and catalytic detoxification of peroxynitrite and hydrogen peroxide [62].

Despite the proven anti-inflammatory effect in macrophages cells, an adverse effect of oral administration of AuNPs against the beneficial probiotic genus Lactobacillus was observed in mice microbiota. At the same time, AuNPs coated by $5 \mathrm{~nm}$ size tannic acid (Au- $5 \mathrm{~nm} / \mathrm{TA}$-treated mice) displayed a lower $\alpha$-diversity of intestinal flora, especially a reduced F/B ratio, the two most abundant phyla in human intestinal microbiota. Similar studies highlighted a markedly decreased species richness of the gut microbiota in mice when AgNPs were orally administrated [28]. Roseburia, Ruminococcaceae, and Odoribacter which are short chain fatty acid (SCFA)-producing bacteria were also reduced in Au-5 nm/TA-treated mice compared to the control. This decrease in the aforementioned bacterial populations, with decreased abundance of Firmicutes species was observed in inflammatory bowel disease (IBD) [63]. The taxonomic Bacteroides phylum increased instead in Au-5 nm/TA-treated mice compared to the control group. The decrease in the abundance of Lactobacillus species responsible for the production of 
hydrogen peroxide leads to imbalances of the normal microbiota. Although, the mechanism by which AuNPs cause the decrease of Lactobacillus probiotics is not known, the potential of AuNPs to induce intestinal dysbiosis is obvious [62].

Contrary to these findings, 4,6-diamino-2-pyrimidinethiol (DAPT)-coated AuNPs applied as therapy for bacterial infection of $E$. coli in a 28 days oral administration trial on mice showed positive effects against bacterial infections with no disturbances of the intestinal microflora [64].

\section{CONCLUSIONS}

A comprehensive understanding of the behavior of nanomaterials when they come into contact with biological fluids and systems is very important to further progress their application in medicine. Sometimes, the balancing between the designed function and desired safety is challenging. This review aimed to explore the stability and chemical reactivity of AgNPs and AuNPs, as well as the evaluation of their persistency in simulated biological fluids, from chemical perspective. In this regard, the dissolution process of $\mathrm{Ag}$ and $\mathrm{Au}$ is strongly influenced by various intrinsic and extrinsic factors, especially $\mathrm{pH}$ and existence of strong binding ligands, being totally different in various biological media. One important aspect is that each study referring to AgNPs should be related to its possible chemical conversion in the experimental medium, in order to provide a more comprehensive understanding of the potential of Ag NPs for further medical applications. The AgNPs and AuNPs can alter the gut microbiota, with serious potential to lead on various diseases. However, some research reported no negative effects for both AgNPs and AuNPs. These contradictory results may be due to the methodological variables, dose, size, coating agent, animal model, methods, the intestinal sampling area, etc. Regarding interaction of AuNPs with the microbiota, there is a lack of data and further systematic research is required to fully assess their potential impact.

\section{ACKNOWLEDGMENTS}

This work was supported by a grant of the Romanian Ministry of Research and Innovation, CCCDI-UEFISCDI, project number PN-III-P2-2.1-PED-20190844, within PNCDI III. 


\section{REFERENCES}

1. J.K. Patra; G. Das; L.F. Fraceto; E.V. Ramos Campos; M.P. Rodriguez-Torres; L.S. Acosta-Torres; L.A. Dias-Torres; R. Grillo; M.P. Swamy; S. Sharma; S. Habtemariam; H.S. Shin; J. Nanobiotechnol., 2018, 16, 71

2. L.V. Stebounova; E. Guio; V.H. Grassian; J. Nanopart. Res., 2011, 13, 233-244

3. Y.N. Slavin; J. Asnis; U.O. Hafeli; H. Bach; J. Nanobiotechnol., 2017, 15, 65

4. M. Auffan; J. Rose; J. Y. Boterro; G.V. Lowry; J.P. Jolivet; M.R. Wiesner; Nat. Nanotechnol., 2009, 4, 634-641

5. K. Khan; I. Khan; Arab. J. Chem., 2019, 12, 908-931

6. A. Pietroiusti; E. Bergamaschi; M. Campagna; L. Campagnolo; G. De Palma; S. Iavicoli; V. Leso; A. Magrini; M. Miragoli; P. Pedata; L. Palombi; I. lavicoli; Part. Fibre Toxicol., 2017, 14, 47

7. W. Utembe; K. Potgieter; A.B. Stefaniak; M. Gulumian; Part. Fibre Toxicol., 2015, 12, 11

8. O. Mbanga; E. Cukrowska; M. Gulumian; J. Nanopart. Res., 2021, 23, 29

9. N. Kamada; S.U. Seo; G.Y. Chen; G. Núñez; Nat. Rev. Immunol., 2013, 13, 321-335

10. E. Thursby; N. Juge; Biochem. J., 2017, 474, 1823-1836

11. R.K. Dudek-Wicher; A. Junka; M. Bartoszewicz; Prz. Gastroenterol., 2018, 13, 85-92

12. B. Lamas; N. Martins Breyner; E. Houdeau; Part. Fibre Toxicol., 2020, 17, 19

13. H.Y. Cheng; M.X. Ning; D.K. Chen; W.T. Ma; Front. Immunol., 2019, 10, 607

14. J. Gao; K. Xu; H. Liu; G. Liu; M. Bai; C. Peng; T. Li; Y. Yin; Front. Cell. Infect. Microbiol., 2018, 6,13

15. L. Peng; Z.R. Li; R.S. Green; I.R. Holzman; J. Lin; J. Nutr., 2009, 139, 16191625

16. A.M. Sheflin; C.L. Melby; F. Carbonero; T.L. Weir; Gut Microbes, 2017, 8, 113-129

17. T. Yatsunenko; F.E. Rey; M.J. Manary; I. Threhan; M.G. Dominguez-Bello; M. Contreras; M. Magris; G. Hidalgo; R.N. Baldassano; A.P. Anokhin; A.C. Heath; B. Warner; J. Reeder; J. Kuczynski; J.G. Caporaso; C.A. Lozupone; C. Lauber; J.C. Clemente; D. Knights; R. Knight; J.I. Gordon; Nature, 2012, 222227

18. S. Carding; K. Verbeke; D.T. Vipond; B.M. Corfe; L.J. Owen; Microb. Ecol. Health Dis., 2015, 26, 26191

19. D. Liang; R.K.K. Leung; W. Guan; W.W. Au; Gut. Pathog., 2018, 10, 3

20. J.E. Belizário; M. Napolitano; Front. Microbiol., 2015, 6, 1050

21. M. Ghebretatios; S. Schaly; S. Prakash; Int. J. Mol. Sci., 2021, 22, 1942 
22. M. Matijašić; T. Meštrović; M. Perić; H. Čipčić Paljetak; M. Panek,; D. Vranešić Bender; D. Ljubas Kelečić; Ž. Krznarić; D. Verbanac; Int. J. Mol. Sci., 2016, 17, 578

23. E. Distrutti; L. Monaldi; P. Ricci; S. Fiorucci; World J. Gastroenterol., 2016, 22, 2219-2241

24. S. Thomas; J. Izard; E. Walsh; K. Batich; P. Chongsathidkiet; G. Clarke; D.A. Sela; A.J. Muller; J.M. Mullin; K. Albert; J.P. Gilligan; K. DiGuilio; R. Dilbarova; W. Alexander; G.C. Prendergast; Cancer Res., 2017, 77, 1783-1812

25. P. Forsythe; W.A. Kunze; Cell. Mol. Life. Sci., 2013, 70, 55-69

26. D.W. Kaufman; J.P. Kelly; G.C. Curhan; T.E. Anderson; S.P. Dretler; G.M. Preminger; D.R. Cave; J. Am. Soc. Nephrol., 2008, 19, 1197-1203

27. M.D. Schulz; C. Atay; J. Heringer; F.K. Romrig; S. Schwitalla; B. Aydin; P.K. Ziegler; J. Varga; W. Reindl; C. Pommerenke; G. Salinas-Riester; A. Böck; C. Alpert; M. Blaut; S.C. Polson; L. Brandl; T. Kirchner; F.R. Greten; S.W. Polson; M.C. Arkan; Nat. Cell. Biol., 2014, 514, 508-512

28. H. Chen; R. Zhao; B. Wang; C. Cai; L. Zheng; H. Wang; M. Wang; H. Ouyang; X. Zhou; Z. Chai; Y. Zhao; W. Feng; Nanolmpact., 2017, 8, 80-88

29. H. Bouwmeester; M. van der Zande; M. A. Jepson; Wiley Interdiscip. Rev. Nanomed. Nanobiotechnol., 2018,10, e1481

30. P. Belteky; A. Ronavari; N. Igaz; B. Szerencses; I.Y. Toth; I. Pfeiffer; M. Kiricsi; Z. Konya; Int. J. Nanomed., 2019, 14, 667-687

31. J.R. Morones; J.L. Elechiguerra; A. Camacho; K. Holt; J.B. Kouri; J.T. Ramirez; M.J. Yacaman; Nanotechnology, 2005, 16, 2346-2353

32. J.M. Zook; S.E. Long; D. Cleveland; C.L.A. Geronimo; R.I. MacCuspie; Anal. Bioanal. Chem., 2011, 401, 1993-2002

33. U.S. EPA; Risk assessment guidance for superfund, Volume I, 1989, Human health evaluation manual (Part A). Interim Final EPA/540/1-89/002. Washington, DC

34. Z. Ferdous; A. Nemmar; Int. J. Mol. Sci., 2020, 21, 2375

35. G.R. Tortella; O. Rubilar; N. Duran; M.C. Diez; M. Martinez; J. Parada; A.B. Seabra; J. Hazard. Mater., 2020, 390, 121974

36. K. Loza; J. Diendorf; C. Sengstock; L. Ruiz-Gonzalez; J.M. Gonzalez-Calbet; M. Vallet-Regi; M. Köller; M. Epple; J. Mater. Chem. B., 2014, 2, 1634-1643

37. Y. Yan; H. Yamg; J. Li; X. Lu; C. Wang; Text. Res. J., 2012, 82, 1422-1429

38. A.B. Stefaniak; M.G. Duling; R.B. Lawrence; T.A. Thomas; R.F. LeBouf; E.E. Wade; M.A. Virji; Int. J. Occup. Environ. Health., 2014, 20, 220-234.

39. M.E. Quadros; R.T. Pierson; N.S. Tulve; R. Willis; K. Rogers; T.A. Thomas; L.C. Marr; Environ. Sci. Technol. 2013, 47, 8894-8901

40. F. Larese-Filon; F. D’Agostin; M. Crosera; G. Adami; N. Renzi; M. Bovenzi; G. Maina; Toxicology, 2009, 255, 33-37

41. W. Zhang; Y. Yao; N. Sullivan; Y. Chen; Environ. Sci. Technol., 2011, 45, 44224428 
42. J. Liu; Z. Wang; F.D. Liu; A.B. Kane; R.H. Hurt; ACS Nano, 2012, 6, 9887-9899

43. Z.M. Xiu; Q.B. Zhang; H.L. Puppala; V.L. Colvin; P.J.J. Alvarez; Nano Lett., 2012, 12, 4271-4275

44. R.A. Sperling; W.J. Park; Phil. Trans. R. Soc. A, 2010, 368, 1333-1383

45. A. Abdelkhaliq; M. van der Zande; A.K. Undas; R.J.B. Peters; H. Bouwmeester; Nanotoxicology, 2020, 14, 111-126

46. N. Hadrup; K. Loeschner; A. Bergström; A. Wilcks; X. Gao; U. Vogel; H.L. Frandsen; E.H. Larsen; H.R. Lam; A. Mortensen; Arch. Toxicol., 2012, 86, 543-551

47. L.A. Wilding; C.M. Bassis; K. Walacavage; S. Hashway; P.R. Leroueil; M. Morishita; A.D. Maynard; M.A. Philbert; I.L. Bergin; Nanotoxicology, 2016, 10, 513-520

48. K. Williams; J. Milner; M.D. Boudreau; K. Gokulan; C.E. Cerniglia; S. Khare; Nanotoxicology, 2015, 9, 279-289

49. C. Cattò; E. Garuglieri; L. Borruso; D. Erba; M.C. Casiraghi; F. Cappitelli; F. Villa; S. Zecchin; R. Zanchi; Environ. Pollut., 2019, 245, 754-763

50. A.B. Javurek; D. Suresh; W.G. Spollen; M.L. Hart; S.A. Hansen; M.R. Ellersieck; N.J. Bivens; S.A. Givan; A. Upendran; R. Kannan; C.S. Rosenfeld; Sci. Rep., 2017, 7, 2822

51. S. van den Brule; J. Ambroise; H. Lecloux; C. Levard; R. Soulas; P.J. De Temmerman; M. Palmai-Pallag; E. Marbaix; D. Lison; Part. Fibre Toxicol., 2015, 13,38

52. C. Kasai; K. Sugimoto; I. Moritani; J. Tanaka; Y. Oya; H. Inoue; M. Tameda; K. Shiraki; M. Ito; Y. Takei; K. Takase; BMC. Gastroenterol., 2015, 15, 100

53. P. Das; J.A.K. McDonald; E.O. Petrof; E. Allen-Vercoe; V.K. Walker; J. Nanomed. Nanotechnol., 2014, 5,235

54. C. Levard; E.M. Hotze; G.V. Lowry; G.E. Brown Jr.; Environ. Sci. Technol., 2012, 46, 6900-6914

55. A.K. Suresh; D.A. Pelletier; W. Wang; J.L. Morrell-Falvey; B. Gu; M.J. Doktycz; Langmuir, 2012, 7, 2727-2735

56. H. Xu; F. Qu; H. Xu; W. Lai; Y.A. Wang; Z.P. Aguilar; H. Wei; Biometals, 2012, $25,45-53$

57. A.L. Bailly; F. Correard; A. Popov; G. Tselikov; F. Chaspoul; R. Appay; A. AlKattan; A.V. Kabashin; D. Braguer; M.A. Esteve; Sci. Rep.-UK., 2019, 9, 12890

58. M.F. Hornos Carneiro; F. Barbosa Jr; J. Toxicol. Env. Heal. B, 2016, 19, 129148

59. E.K. Breitner; S.M. Hussain; K.K. Comfort; J. Nanobiotechnol., 2015, 13, 56

60. M.M. Arnida; A. Ray; C.M. Peterson; H. Ghandehari; Eur. J Pharm. Biopharm., 2011, 77, 417-423

61. S.J. Berners-Price; A. Filipovska; Metallomics. 2011, 3, 863-873

62. S. Zhu; X. Jiang; M.D. Boudreau; G. Feng; Y. Miao; S. Dong; H. Wu; M. Zeng; J.J. Yin; J. Nanobiotechnol., 2018, 16, 86. 
ZAMFIRA DINCA, TEODORA MOCAN, LACRIMIOARA SENILA, OANA CADAR

63. I. Khan; N. Ullah; L. Zha; Y. Bai; A. Khan; T. Zhao; T. Che; C. Zhang; Pathogens, 2019, 8, 126

64. J. Li; R. Cha; X. Zhao; H. Guo; H. Luo; M. Wang; F. Zhou; X. Jiang; ACS Nano, 2019, 13, 5002-5014 